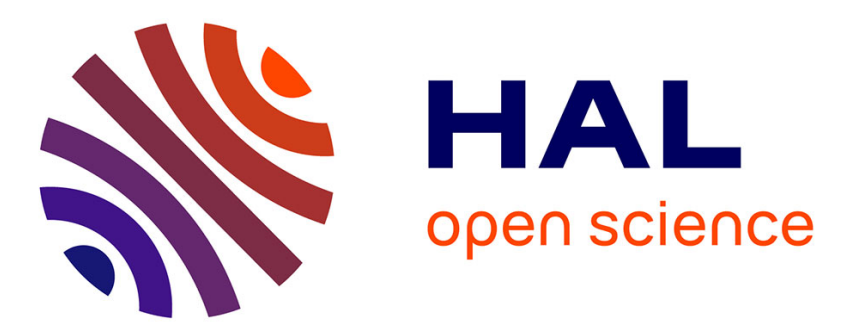

\title{
Notes forestières sur la Côte française des Somalis. le Harrar et le Choa
}

\author{
Louis Duplaquet
}

\section{To cite this version:}

Louis Duplaquet. Notes forestières sur la Côte française des Somalis. le Harrar et le Choa. Revue forestière française, 1953, 5, pp.347-351. 10.4267/2042/26886 . hal-03379882

\section{HAL Id: hal-03379882 \\ https://hal.science/hal-03379882}

Submitted on 15 Oct 2021

HAL is a multi-disciplinary open access archive for the deposit and dissemination of scientific research documents, whether they are published or not. The documents may come from teaching and research institutions in France or abroad, or from public or private research centers.
L'archive ouverte pluridisciplinaire HAL, est destinée au dépôt et à la diffusion de documents scientifiques de niveau recherche, publiés ou non, émanant des établissements d'enseignement et de recherche français ou étrangers, des laboratoires publics ou privés. 


\section{NOTES FORESTIẼRES SUR LA COTE FRANÇAISE DES SOMALIS, LE HARRAR ET LE CHOA}

Dans son $\mathrm{n}^{\circ}$ de janvier I953 (p. 62), la Revue Forestic̀re Française a rendu brièvement compte d'un article de E.-H.-F. SwaIN paru dans Unasylva de mars I952 et intitulé "Vers une foresterie éthiopienne? ». traduction un peu libre de " Forestry Possibilities in Ethiopia »).

Ayant eu l'occasion de rencentrer M. Swain à Addis-Abeba en octobre 1952, nous nous proposons d'emprunter plus largement à son travail. Mais auparavant, nous désirerions dire un mot de la Côte Française des Somalis, qui constitue en quelque sorte la porte d'entrée de l'Empire du Négus.

\section{Côte Française des Somalis}

Les nombreux voyageurs qui font escale à Djibouti, où règne une température accablante, s'imaginent volontiers que l'hinterland n'est qu'un désert qu'aucune végétation n'est capable de peupler. Dans la ville même, le trop fameux palmier en zinc, qui n'a peut-être jamais existé, n'est plus connu aujourd'hui que par le café-bar qui porte son nom. Les rues sont bordées de laurier du Yémen d'une belle taille et de jolis jardins entourent les coquettes villas. Il faut reconnaitre que cette transformation n'a pu s'accomplir qu'à l'aide de l'eau apportée aux plantes.

Quant au territoire de la Côte Française des Somalis, qui parait. minuscule sur les cartes que l'on a coutume de consulter, il se présente sensiblement sous la forme d'un cercle de près de $100 \mathrm{~km}$ de rayon, dont une bonne partie, il est vrai, est occupée par le Golfe de Tadjoura. Il y existe de réritables déserts relativement peu étendus, mais dès qu'on s'élève au-dessus du niveau de la mer, la température s'abaisse sensiblement et une végétation d"épineux fait son apparition. La montagne n'est pas loin; il nous a été donné de visiter à I $200 \mathrm{~m}$ d'altitude et à une cinquantaine de kilomètres de Djibouti à̀ vol d'oiseau, la forêt du Daï, constituée en Parc National par arrêté du Gouverneur.

Cette forêt est peuplée essentiellement de 3 essences:

$\mathrm{I}^{\circ}$ la plus abondante, un buis;

$2^{\circ}$ la plus intéressante, Juniperus procera;

$3^{\circ}$ enfin l'olivier sauvage. 
Le buis devient un arbre de futaie de 0,30 à $0,35 \mathrm{~m}$ de diamètre.

Le genévrier atteint de très belles dimenșions. Malheureusement, il dépérit au Dai de façon inquiétante et on se demande pourquoi. S'il s'y rencontre encore d'assez nombreux sujets anciens, dont les branches sèchent peu à peu, on n'y voit pas de jeunesse. Peut-être y aurait-il lieu, pour favoriser la régénération, d'effectuer des crochetages sous les porte-graines. Au lieu de ces soins, lorsque nous nous $\mathrm{y}$ trouvions, $\mathrm{y}$ campait en même temps que nous un peloton méhariste, et l'on sait les dégâts que peuvent causer des chameaux en forêt !

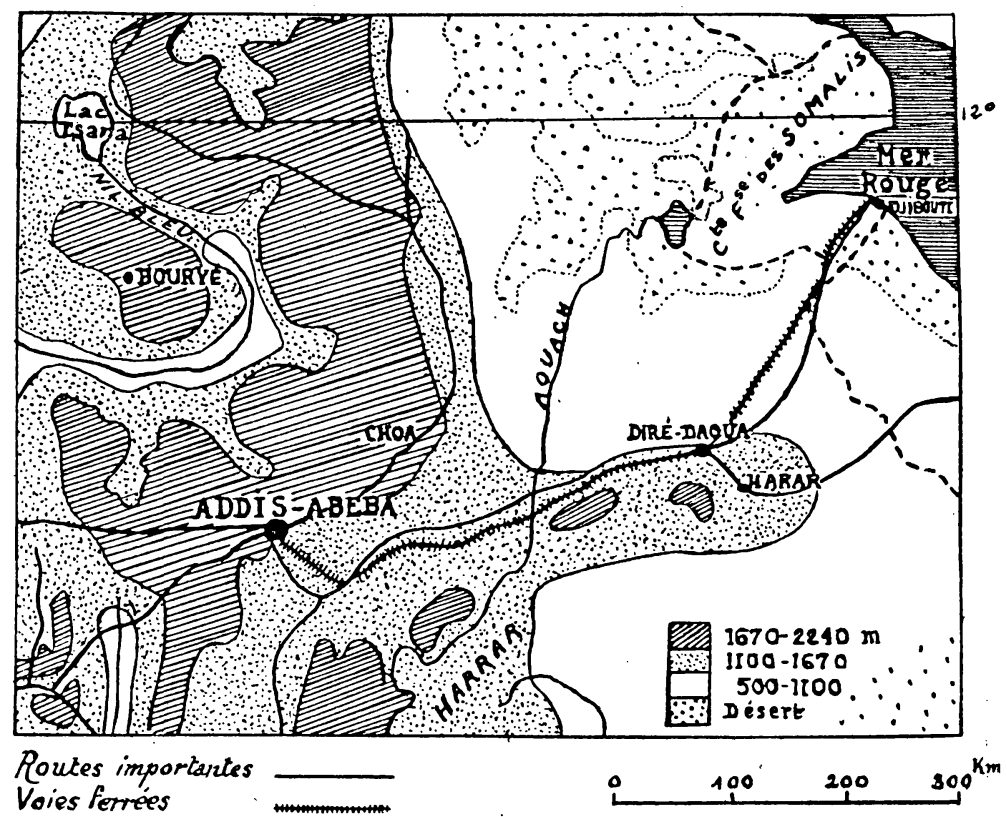

Cóte Française des Somalis, Harrar et Choa.

Voici ce qu'écrit Swaln du comportement de cette espèce en Ethiopie: "Juniperus procera peut supporter 5 mois de sécheresse " par an, sur l'épine dorsale au sol mince et glacé de l'Ethiopie, et " endurer mille années d'adversité, pour faire croître un vétéran " de $3 \mathrm{~m}$ de diamètre à hauteur d'homme ". Mais un peu plus loin, il reconnait aussi que $J$. procera peut " atteindre les confins du désert ». Il s'agit d'un arbre extrêmement plastique, susceptỉble de rendre de grands services malgré la lenteur relative de sa croissance, estimée à $2,5 \mathrm{~mm}$ de diamètre par an.

\section{HARRAR}

Le massif de l'Harrar est séparé de celui du Choà et du pays Amhara proprement dit par la dépression de l'Aouach et des lacs 
éthiopiens. La voie ferrée franco-éthiopienne de Djibouti à AddisAbeba le longe sur une bonne partie de son parcours et c'est sur ses contreforts, à Diré-Daoua, que réside l'état-major français du chemin de fer (I).

Si l'on cherche à gagner la ville de Harrar, située à I 936 m d'alt. et où plane encore le souvenir de Rimbaud, on rencontre quelques spécimens de Juniperus procera et, cultivé en banquettes, le Kât (Catha edulis), de la famille des Célastracées, dont les feuilles, utilisées comme stupéfiant, donnent lieu à un commerce intense. L'avion-cargo, qui nous a ramené d'Addis-Abeba à Djibouti, a chargé à Diré-Daoua à destination d'Aden, une quantité impressionnante de sacs bourrés de ces feuilles. $\mathrm{Si}$, au point de vue de la santé publique, le Kât est désastreux, sa culture sur banquettes dans les montagnes du Harrar présente, en ce qui concerne la défense et la conservation des sols, un intérêt certain en s'opposant à l'érosion des pentes.

On rencontre aussi les premiers boisements artificiels d'eucalyptus, dont il sera parlé plus loin à propos du Choa.

\section{CHOA}

Lorsque, après une nuit de train, ayant quitté la veille au soir la vallée semi-désertique de l'Aouach, on se réveille au petit matin sur les hauts plateaux du Choa, on est surpris par la différence de température d'abord, l'aspect du pays ensuite. Une terre noire et riche porte des champs de blé et, au loin, on aperçoit les eucalyptus d'Addis-Abeba.

C'est qu'en effet, ainsi que le dit E. Berlan, " l'eucalyptus est " l'arbre d'Addis-Abeba. Dans cette ville qui s'étale sur plus de (" $50 \mathrm{~km}^{2}$, les divers quartiers sont parfois séparés par une forêt " dense. Ióo oqo habitants vivent sous ro 000 ooo d'arbres... Cette " forêt odorante et légèrement embrumée fait les matins bleus " d'Addis-Abeba ").

Le fait que c'est un de nos compatriotes, le forestier MondonVIDALHET, conseiller de MÉNÉLIK, qui le premier rapporta d'Australie les précieuses graines vers I894 et introduisit l'eucalyptus au Choa, est pour nous Français un sujet de légitime fierté.. " L'une de ces espèces, écrit encore SwaIn, était un arbre robuste venant " des âpres collines australiennes, suffisamment plastique pour sup“ porter à la fois les pluies d'hiver et d'été, aussi bien que la neige. " Il manifesta une croissance prodigieuse. On en planta 20000 par " hectare, et l'on procéda à des coupes d'éclaircies dans les qua" trième, septième, quinzième et vingt-cinquième années. On laissa " 800 arbres par ha, représentant un volume de $570 \mathrm{~m}^{3}$ à vingt-six " ans et $\mathrm{I} 080 \mathrm{~m}^{3}$ à quarante ans ". L'espèce en question est $E$. globulus et nous n'en avons pas aperçu d'autres. Il paraît regretta-

(I) Nous avons eu la joie d'y trouver un fils de M, l'Inspecteur Général Turc. 
ble que, depuis l'introduction déjà lointaine des premiers eucalyptus, on n'ait pas cherché à expérimenter d'autres espèces du même genre, car les produits d'E. globulus ne sont pas les plus appréciés. En fait, son bois n'est guère utilisé que pour le chauffage et la construction des toukouls, habitations rurales faites de clayonnages revêtus d'argile.

Aujourd'hui le Bahaar zarf (arbre étrange venu d'au delà des mers), prospère non seulement dans la capitale même, située à 2445 $\mathrm{m}$ d'alt., mais aussi sur les collines basaltiques environnantes qui s'élèvent jusqu'à plus de $3.000 \mathrm{~m}$.

Un bois très apprécié par les ateliers du chemin de fer est celui du Zigba, qui n'est autre que le conifère Podocarpus gracilior. Nous n'en avons pas vu sur pied, mais voici ce qu'en dit Swarn: "Podo“ carpus gracilior est inféodé à un monde différent (de Juniperus ( procera). Il ne peut escalader les hauteurs escarpées des collines ( arides, ni atteindre les confins du désert; il ne peut pas non plus ( ' s'étendre aussi loin vers le Nord ou'le Sud. Il a une vitesse de ( croissance presque aussi lente, mais une moindre résistance à la ( sécheresse. Il recherche des stations plus clémentes et la compa" gnie d'un mélange de feuillus moins robustes. Il trouve sa place " dans des vallées abritées d'une altitude moins élevée, où il est re" joint par les plus aventureux des arbres feuillus des régimes à ( courtes périodes de sécheresse ).

\section{GIBIER}

L'Ethiopie est l'un des pays les plus giboyeux du monde, surtout si l'on considère qu'il n'y existe pas de grandes réserves de chasse comme dans les territoires britanniques ou même les possessions françaises.

Les grands fauves y sont légion et l'emblème national du lion de Juda n'est pas surfait. La panthère, le guépard si gracieux et si facile à apprivoiser, sont fréquents. La hyène vient hanter la nuit les murailles de la vieille citadelle de Harrar. Le lycaon, le chacal, le lynx caracal trouvent à se nourrir.

- A peu près toutes les antilopes existent, depuis le Grand Koudov et l'Elan de Derby jusqu'à la petite antilope Dig-dig.

$\mathrm{La}$ route est parfois barrée par d'immenses bandes de singes cynocéphales.

Parmi les gros oiseaux, on rencontre gypaëtes, vautours, milans, outardes, etc...

\section{Conclusions}

On ne peut s'empêcher de regretter que l'Ethiopie ne possède pas de Service forestier organisé, car les possibilités forestières y sont très grandes et la tâche à accomplir devrait être passionnante. 
La solution des missions F.A.O., auxquelles appartient M. SwaIn, ancien forestier d'Australie, ne parait pas être la meilleure dans ce pays, Il serait souhaitable que le gouvernement impérial accepte le détachement de quelques jeunes forestiers français pleins d'allant pour continuer l'œuvre de Mondon-Vidalhet.

Il semble (ce que nous a confirmé l'ambassade de France à AddisAbeba) que le moment ne serait pas mal choisi. Nous avons actuellement " la cote " au pays de la reine de Saba et du mystérieux prêtre Jean. Si l'anglais est la seconde langue officielle (la première étant bien entendu l'amharite), le français est plus volontiers parlé par les Ethiopiens lettrés, au nombre desquels il y a lieu de citer en tout premier lieu Sa Majesté Hailé Sélassié elle-même, qui manie la langue de Racine avec beaucoup plus d'aisance que celle de Shakespeare.

Nous ne pouvons que souhaiter que cet appel soit entendu, tant du gouvernement éthiopien que de camarades décidés à séjourner et à travailler dans cette région si attachante du continent africain où ils retrouveront de nombreux et sympathiques compatriotes.

\section{BIBLIOGRAPHIE}

L. Duplaquet.

E. Berlan. - L'eucalyptus à Addis-Abeba et au Choa. Revue de Géographie Alpine, r95I, fasc. III, p. $57 \mathrm{I}$.

E.-H.-F. Swain. - Forestry Possibilities in Ethiopia. Unasylva, vol, VI, $\mathrm{n}^{\bullet} \mathrm{I}$, mars 1952.

\section{A propos de la croissance de l'Abies grandis}

Nos lecteurs ont appris par le numéro de janvier I953 les très graves dommages subis par l'Arboretum des Barres, lors de la tempête du I3 décémbre 1952.

Nots y indiquions en particulier que l'Abies grandis dont la cime dépassait tous les arbres du domainic et était visible à plusieurs kilomètres à la ronde, avait été renversé.

On a déjà signalé ici (Rev. For. Franç., I95I, p. 40r) et ailleurs (Rev. Eaux Forêts, tome 81, 1943, p. 352), les belles dimensions atteintes par des arbres de la même espèce:

A Rumilly (Haute-Savoie), un arbre exceptionnel atteignait à 35 ans les dimensions suivantes:

Circonférence à I,30 $\mathrm{m}: 2,74 \mathrm{~m}$; hauteur totale: $28 \mathrm{~m}$; volume: $10 \mathrm{~m}^{3}$.

A Bulgnéville (Vosges) sur infralias, un arbre planté en r 886 mesurait: en 1925 (40 ans) : $1,85 \mathrm{~m}$ sur $30 \mathrm{~m}$,

en 1942 ( 57 ans) $: 2,33 \mathrm{~m}$ avec une hauteur utile de $24 \mathrm{~m}$ et un volume estimé de $5,5 \mathrm{~m}^{3}$.

L'arbre des Barres (Pelouse 1o de l'Arboretum) avait 76 ans; sa longueur totale était de 42 mètres, sa longueur utile de $30,30 \mathrm{~m}$ (i1 était mutiflèche ensuite).

Les circonférences étaient respectivement les suivantes: $\grave{a} I, 30 \mathrm{~m}: 3,93 \mathrm{~m}$;

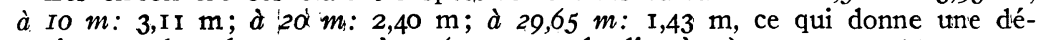
croissance de $9,6 \mathrm{~cm}$ par mètre $(3 \mathrm{~cm}$ sur le diamètre) sur $30 \mathrm{~m}$.

Le volume total était de $18,65 \mathrm{~m}^{3}$, dont $\mathrm{I} 8,20 \mathrm{~m}^{3}$ pour la grume découpée à $30 \mathrm{~m}$.

J. P. 